\title{
Implanted impurities in wide band gap semiconductors
}

\author{
P. Kessler ${ }^{1, a}$, K. Lorenz $z^{2, b}$ and R. Vianden ${ }^{1, c}$ \\ ${ }^{1}$ Helmholtz-Institut für Strahlen- und Kernphysik \\ Nussallee 14-16, 53115 Bonn, Germany \\ ${ }^{2}$ ITN, Estrada Nacional 10, P-2686-953 Sacavem, Portugal \\ akessler@hiskp.uni-bonn.de, 'lorenz@itn.pt, cvianden@hiskp.uni-bonn.de
}

\begin{abstract}
Keywords: PAC - GaN - ZnO - Nitride Semicoductors - II-VI Compound - Annealing Behavior
\end{abstract}
\begin{abstract}
Wide band gap semiconductors, mainly GaN, have experienced much attention due to their application in photonic devices and high-power or high-temperature electronic devices. Especially the synthesis of $\operatorname{In}_{\mathrm{x}} \mathrm{Ga}_{1-\mathrm{x}} \mathrm{N}$ alloys has been studied extensively because of their use in LEDs and laser diodes. Here, In is added during the growth process and devices are already very successful on a commercial scale. Indium in nitride ternary and quaternary alloys plays a special role; however, the mechanisms leading to more efficient light emission in In-containing nitrides are still under debate. Therefore, the behavior of In in $\mathrm{GaN}$ and $\mathrm{AlN}$, the nitride semiconductor with the largest bandgap is an important field of study. In is also an important impurity in another wide band gap semiconductor - the II-VI compound $\mathrm{ZnO}$ where it acts as an n-type dopant. In this context the perturbed angular correlation technique using implantation of the probe ${ }^{111}$ In is a unique tool to study the immediate lattice environment of In in the wurtzite lattice of these wide band gap semiconductors. For the production of $\mathrm{GaN}$ and $\mathrm{ZnO}$ based electronic circuits one would normally apply the ion implantation technique, which is the most widely used method for selective area doping of semiconductors like Si and GaAs. However, this technique suffers from the fact that it invariably produces severe lattice damage in the implanted region, which in nitride semiconductors has been found to be very difficult to recover by annealing. The perturbed angular correlation technique is employed to monitor the damage recovery around implanted atoms and the properties of hitherto known impurity - defect complexes will be described and compared to proposed structure models.
\end{abstract}

\section{Introduction}

The immense success of wide band gap semiconductor devices in the field of light emitting diodes, laser and other optoelectronic devices in recent years has led to greatly improved base material mainly for GaN and AIN. Similarly ZnO, applicable for UV devices and as ITO (Indium Tin Oxide) substitute, has become available in superior quality. The basic parameters of the materials discussed here can be found in Table 1.

It therefore seems probable that even more fields of application for these wide band gap materials, which open the road to high temperature, high power devices, will become important. Further, the radiation hardness and the possibility to tailor the band gap by forming ternary alloys like GaInN, AlInN or ZnCdO are advantageous for terrestrial and space photovoltaics.

However, all more sophisticated devices usually require lateral structuring which in standard semiconductor technology is achieved in a controlled and reproducible manner by ion implantation. Therefore, it will be important to understand and control the production and annealing of defects, which are unavoidable during ion implantation as well as the interaction of implanted dopant atoms with these defects. The perturbed angular correlation (PAC) technique, described by Zacate and Jaeger in the present volume [1], is a unique tool to investigate the lattice surrounding of implanted impurities and the fact that with ${ }^{111} \operatorname{In}\left({ }^{111} \mathrm{Cd}\right)$ a PAC-probe is available where the parent is: 
Table 1: Crystal parameters and properties of group III nitrides and $\mathrm{ZnO}$ at $300 \mathrm{~K}$ taken from ref. [2] if not indicated otherwise in the table.

\begin{tabular}{|l|l|l|l|l|}
\hline & AlN & GaN & InN & ZnO \\
\hline Lattice constant a $[\AA]$ & 3.112 & 3.189 & 3.533 & $3.2495[6]$ \\
\hline Lattice constant c $[\AA]$ & 4.982 & 5.185 & 5.693 & $5.2069[6]$ \\
\hline c/a ratio (ideal $\sqrt{8 / 3}=1.633)$ & 1.601 & 1.626 & 1.611 & $1.6(1)[3,4,5]$ \\
\hline u-Parameter (ideal 3/8 =0.375) & 0.382 & 0.377 & 0.379 & $0.38[3,4,5]$ \\
\hline Band gap [eV] & 6.2 & 3.4 & $0.7-1.9^{*}$ & $3.4[7]$ \\
\hline Melting point [K] & 3273 & 2773 & 1373 & 2248 \\
\hline Density [g/cm $\left.{ }^{3}\right]$ & 3.23 & 6.15 & 6.81 & $5.605[6]$ \\
\hline $\begin{array}{l}\text { Thermal expansion coefficient in } \\
\text { a }\left[\mathrm{K}^{-1}\right]\end{array}$ & $4.2 \cdot 10^{-6}$ & $5.6 \cdot 10^{-6}$ & $3.8 \cdot 10^{-6}$ & $4.31 \cdot 10^{-6}[7]$ \\
\hline $\begin{array}{l}\text { Thermal expansion coefficient in } \\
\mathrm{c}\left[\mathrm{K}^{-1}\right]\end{array}$ & $5.3 \cdot 10^{-6}$ & $3.2 \cdot 10^{-6}$ & $2.9 \cdot 10^{-6}$ & $2.49 \cdot 10^{-6}[7]$ \\
\hline$*$ still under debate in the literature. & & & \\
\hline
\end{tabular}

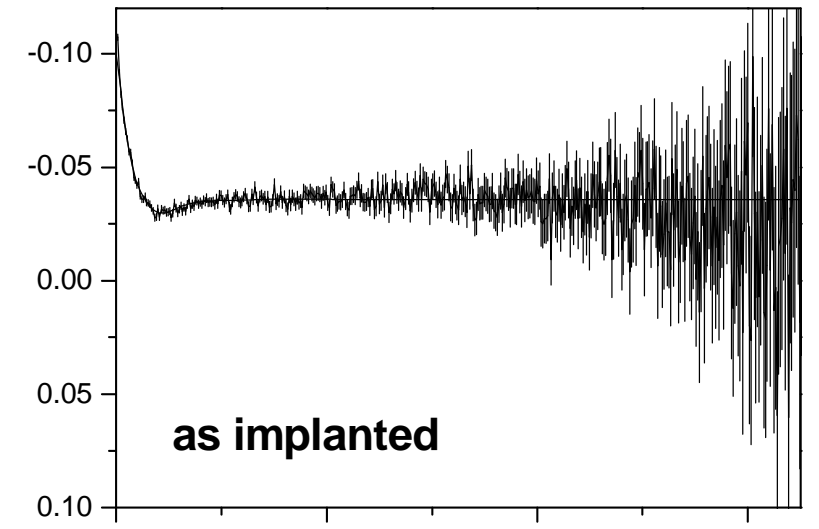

$\mathbf{R}(\mathbf{t})$

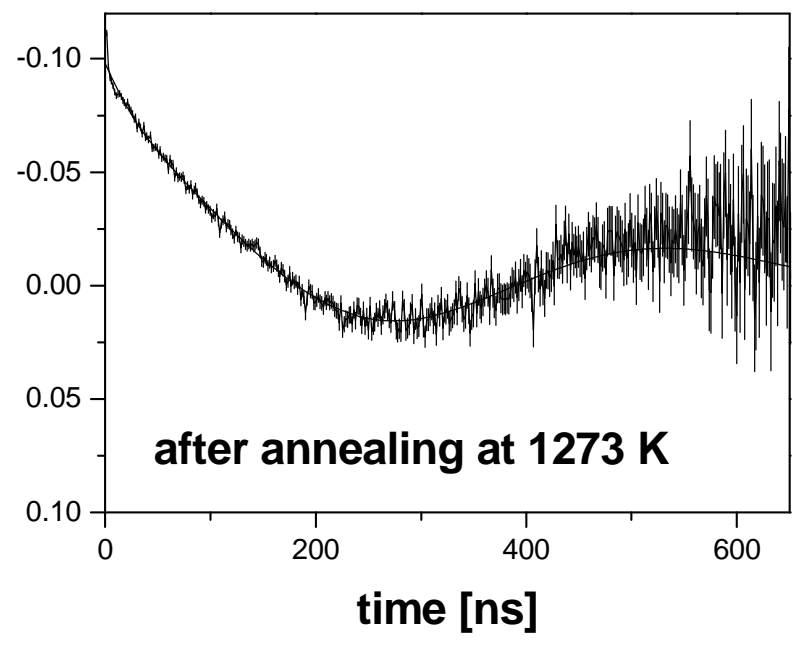

Fig. 1: Typical PAC spectra (counting rate ratio $\mathrm{R}(\mathrm{t})$ [1] obtained with ${ }^{111} \mathrm{In}$ after implantation into $\mathrm{GaN}$ (top) and after annealing for $10 \mathrm{~min}$ at $1273 \mathrm{~K}$ under $\mathrm{N}_{2}$ atmosphere (bottom). 
a native constituent of many ternary nitride semiconductors and the daughter isoelectronic to $\mathrm{Zn}$ in $\mathrm{ZnO}$, makes the results of these studies even more relevant.

Further, up to now it is still unclear what is the reason of the astonishing large quantum efficiency of many nitride based optical devices containing In. Various mechanisms have been suggested to explain this puzzle. Indium rich nanoclusters [8], local bandgap variations due to compositional [9] or strain [10] inhomogeneities as well as microstructures [11] have been proposed to be the origin of exciton localization at radiative traps preventing them from diffusing to nonradiative recombination centers [12]. It is interesting to note that in all cases Indium plays an important role. Recently, it was shown by Extended X-ray Absorption Fine Structure (EXAFS) that non-random aggregation of In atoms occurs in InGaN epilayers [13]. The degree of phase segregation was shown to decrease with increasing InN concentration. Since this phenomenon is accompanied by a decrease of luminescence efficiency these regions of weak phase segregation were proposed to act as efficient localization centre for excitons. Unfortunately up to now a convincing proof for any of the suggestions is lacking. Here it will be shown that the results of PAC studies using the ${ }^{111}$ In PAC probe might help to solve the puzzle.

\section{Annealing Studies}

First studies of the recovery of implantation-induced damage using PAC in GaN were carried out as soon as material of acceptable quality became available [14, 15, 16, 17]. Naturally, in these experiments the probe ${ }^{111}$ In was employed, on the one hand because it is commercially available but on the other, more important, because for practical applications mainly ternary alloys like GaInN are used. Implantation of In would therefore allow the formation of regions with different bandgaps on one wafer, for instance.

The PAC spectra obtained immediately after implantation showed a shape typical for probes in largely disordered lattice environment (Fig. 1 top). Annealing at various temperatures up to $1273 \mathrm{~K}$ led to a gradual recovery of the crystal structure, resulting in a small quadrupole interaction frequency of $v_{Q}=6.3(1) \mathrm{MHz}$ (Fig.1 bottom) [18, 16]. It is due to the wurtzite structure which is the thermodynamically stable structure of $\mathrm{GaN}$ as well as of all the other materials discussed in this review (Fig. 2). It can be described by two simple hexagonal lattices which are characterized by the c/a ratio and consist of $\mathrm{N}(\mathrm{O}$ in the case of $\mathrm{ZnO})$ and a group III element ( $\mathrm{Zn}$ in the case of $\mathrm{ZnO})$, respectively. The lattices are shifted relative to each other along the hexagonal $c$ axis as quantified by the so called u-parameter. Since the distribution of charges in this structure is not cubic an axially symmetric electric field gradient is expected at the lattice sites with its principal component pointing along the c-axis.

However, it soon became obvious that the fraction of In probes on regular lattice sites did only reach values of app. $60 \%$ (Fig. 3). This prompted various studies where the annealing temperature, duration and atmosphere were varied $[14,15,16,17,18]$. But in none of these studies a larger substitutional fraction could be achieved.

A similar result was found for In in $\operatorname{AlN}[19,20]$. Here maximum substitutional fractions of app. 50\% were found under the best annealing conditions. The corresponding quadrupole interaction frequency (QIF) has a value of $v_{Q} \approx 30 \mathrm{MHz}$. However, unlike in GaN the remaining fraction of probe atoms is not highly disturbed but shows a well defined large QIF of $v_{Q} \approx 300 \mathrm{MHz}$.

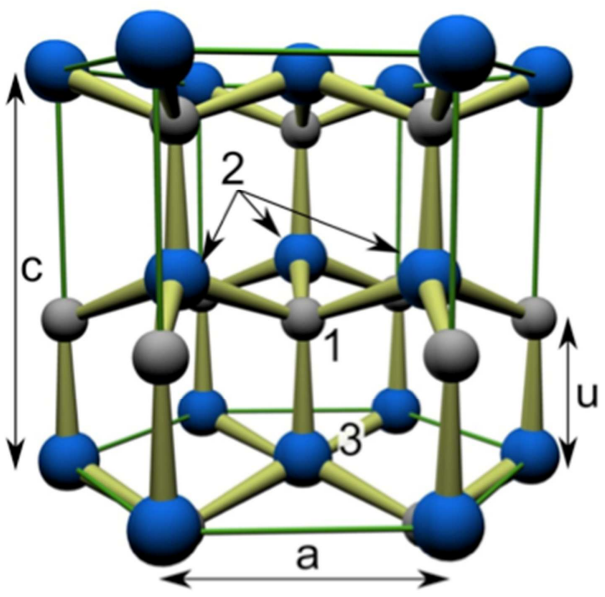

Fig. 2: Model of the wurtzite lattice. The cation and substitutional probe atoms occupy position 1. The three equivalent $\mathrm{NN}$ anion positions in the (0001) plane are labelled 2 and the anion site in <0001> direction is labelled 3 . 


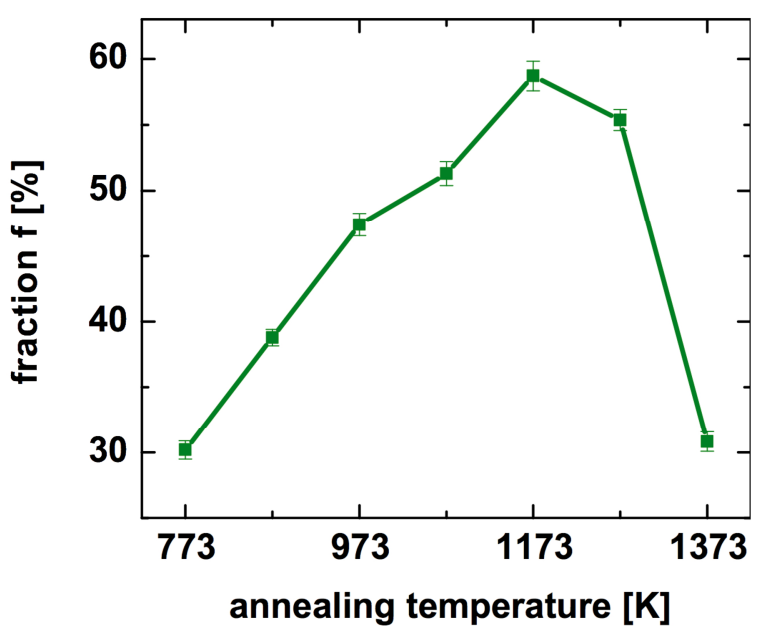

Fig. 3: Fraction of ${ }^{111}$ In probes on regular GaN lattice sites (Pos. 1 in Fig. 2) after annealing for $10 \mathrm{~min}$ at different temperatures under $\mathrm{N}_{2}$ atmosphere.
In order to exclude the possibility that two different lattice sites are populated Rutherford Backscattering/Channelling (RBS/C) measurements were carried out on AlN implanted with $3^{*} 10^{14} \mathrm{In} / \mathrm{cm}^{2}$ and annealed under conditions where the PAC data had yielded a maximum substitutional fraction [19]. With a $1 \mathrm{~mm}$ diameter collimated beam of $2 \mathrm{MeV} \mathrm{He}$ ions full angular scans were recorded across the <0001> and $\langle\bar{S} 113\rangle$ axial directions, using a two-axes goniometer (Fig. 4). The almost complete overlap of scans for $\mathrm{Al}$ and In show that In is incorporated preferentially on substitutional Al-sites. Simulations of ion channelling with the Monte Carlo program package FLUX [21] were then used to fit the angular scans. As a result of this analysis it was shown that In is incorporated slightly displaced from the substitutional Al-site with displacements of $\sim 0.05 \AA$ and $\sim 0.1 \AA$ perpendicular to the $<0001>$ and the $\langle\overline{2} 113>$ axis, respectively. The substitutional fraction $\mathrm{f}_{\mathrm{s}}$ can be estimated to be $\sim 90 \%$ by $\mathrm{f}_{\mathrm{s}}=\left(1-\chi_{\min }(\mathrm{In}) /\left(1-\chi_{\min }(\mathrm{Al})\right)\right.$ where $\chi_{\min }(\mathrm{In} / \mathrm{Al})$ are the minimum yields of In and $\mathrm{Al}$.

Therefore the fast frequency observed in PAC measurements is attributed to a fraction of substitutional In probes that trap a next point defect, as it will be discussed in detail later.

Implantation and annealing studies in $\mathrm{InN}$ are quite scarce, mainly due to the lack of material with sufficiently high crystalline quality. At room temperature Dogra et al. [22] observed a QIF of $v_{Q}=28 \mathrm{MHz}$ at the cation site in $\mathrm{InN}$ which is consistent with measurements for other nitrides. However, it was not possible to anneal the implantation damage completely, which is attributed to inherent defects in the InN lattice. Additionally, a sizable fraction of In probes was found in an In metal environment. This is most probably due to In probes implanted into In metal droplets formed on the surface of the samples during the epitaxial growth.

Besides In, the behavior of implanted Hf was investigated in GaN and AlN [16, 19]. Surprisingly, for $\mathrm{GaN}$ it was found that after annealing nearly $80 \%$ of the probe ${ }^{181} \mathrm{Hf}$ showed a unique QIF indicating that it is substitutionally incorporated into these lattices. Additional RBS/Channelling lattice location measurements confirmed that Hf occupies exclusively the cation sites in both GaN and AlN.

The incorporation of ${ }^{111} \mathrm{In}$ diffused into $\mathrm{ZnO}$ powder samples and its interaction with defects created by the introduction of excess $\mathrm{Zn}$ has been studied already by Forkel [23] and Wolf

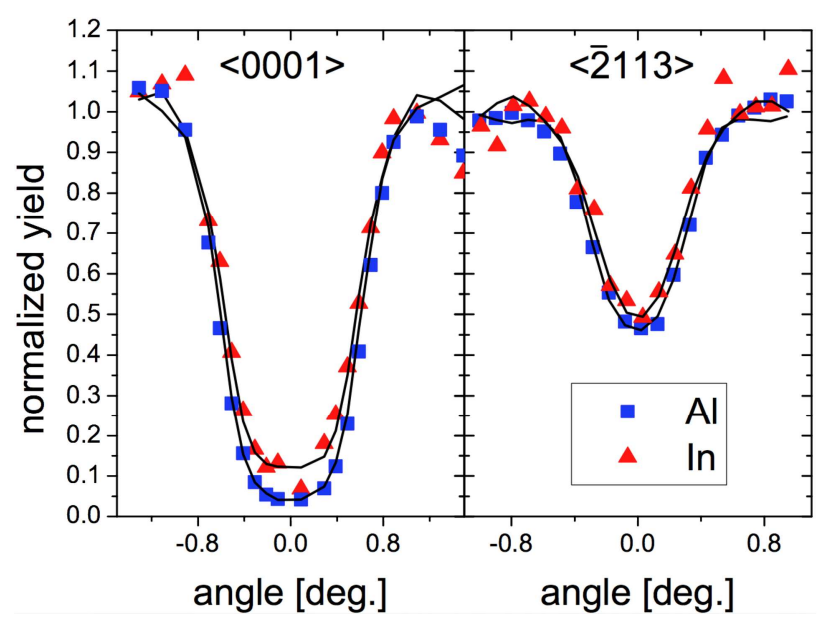

Fig. 4: RBS/C scans across the $<0001>$ and $<\overline{2} 113>$ axes for depth windows on the In and $\mathrm{Al}$ signal comprising the entire implanted region for a sample implanted with $3 \times 10^{14}$ at $/ \mathrm{cm}^{2}$ In at $160 \mathrm{keV}$. The Monte Carlo simulation of the scans are also shown (solid lines). 
Table 2: Electric field gradient $V_{z z}^{\text {exp }}$ measured at $293 \mathrm{~K}$ for various probe atoms at the cation site of wide band gap semiconductors. The values are obtained by dividing by the appropriate quadrupole moment and the Sternheimer antishielding factor given in the table. References are given in square brackets in the table.

\begin{tabular}{|c|c|c|c|c|c|c|}
\hline Probe & $|\mathbf{Q M}|$ & $\left(1-\gamma_{\infty}\right)$ & GaN & AlN & InN & $\mathrm{ZnO}$ \\
\hline & {$\left[10^{-24} \mathrm{~cm}^{2}\right]$} & {$[24,25]$} & \multicolumn{4}{|c|}{$V_{z z}^{\exp }\left[10^{15} \mathrm{~V} / \mathrm{cm}^{2}\right]$} \\
\hline${ }^{77} \mathrm{Br}(\mathrm{Se})$ & $0.76(8)[26]$ & $98[27]$ & - & - & - & $22.7(30)$ \\
\hline${ }^{111} \operatorname{In}(\mathrm{Cd})$ & $0.83(13)[28]$ & 30 & $1.02(5)[29]$ & 4.8(1) [19] & $3.72(3)$ & $5.04(8)[15]$ \\
\hline${ }^{111 \mathrm{~m}} \mathrm{Cd}(\mathrm{Cd})$ & $0.83(13)$ & 30 & $1.0(1)[15]$ & $4.8(1)$ & - & $5.02(8)$ \\
\hline${ }^{111} \mathrm{Ag}(\mathrm{Cd})$ & $0.83(13)$ & 30 & - & - & - & $5.3(-)[40]$ \\
\hline${ }^{117} \mathrm{Cd}(\mathrm{In})$ & $-0.64(4)[30]$ & 26 & $4.9(-)[31]$ & $10.1(-)$ & - & $4.85(1)[41]$ \\
\hline${ }^{181} \mathrm{Hf}(\mathrm{Ta})$ & $2.36(5)[32]$ & 62 & $9.8(1)[33]$ & $16.0(4)$ & - & $5.8(1)[43]$ \\
\hline${ }^{172} \mathrm{Lu}(\mathrm{Yb})$ & $2.87(41)[30]$ & 80 & $5.9(-)$ [34] & - & - & $5.7(1)[43]$ \\
\hline${ }^{69} \mathrm{Ga}$ (stable) & $+0.168[30]$ & 11 & $6.41(3)[52]$ & & & \\
\hline${ }^{27} \mathrm{Al}$ (stable) & $+0.140(2)[30]$ & 3.5 & & 18.4(-) [35] & & \\
\hline${ }^{115}$ In (stable) & $+0.861[30]$ & 26 & & & $0.64(-)[36]$ & \\
\hline${ }^{67} \mathrm{Zn}$ (stable) & $+0.150[30]$ & 13.3 & & & & $5.0(-)[52]$ \\
\hline
\end{tabular}

et al. [37]. Recent measurements with implanted ${ }^{111}$ In in high quality single crystals essentially confirm these earlier results. In contrast to the nitride semiconductors, after annealing for 2 min in nitrogen flow at $1300 \mathrm{~K}, \approx 100 \%$ of the probes show a unique QIF of $v_{Q}=31 \mathrm{MHz}$ (Fig. 5), which can be associated with In at substitutional $\mathrm{Zn}$ sites [38]. Recent annealing studies of ${ }^{111}$ In [39] recoil implanted into $\mathrm{ZnO}$ show similar results.

Further experiments were carried out with the probes ${ }^{111} \mathrm{Ag}[40],{ }^{117} \mathrm{Cd}[41],{ }^{77} \mathrm{Br}[42],{ }^{111 \mathrm{~m}} \mathrm{Cd}$ [41], ${ }^{171} \mathrm{Lu}$ [43], and ${ }^{181} \mathrm{Hf}$ [18] (Fig. 5.) In all cases the PAC results indicate a recovery of the lattice damage and an incorporation of the probes onto unique, most probable substitutional $\mathrm{Zn}$ sites. The values derived for the lattice EFG at $293 \mathrm{~K}$ for all materials studied are collected in Table 2.

\section{Temperature dependence of the EFG}

Measurements of the EFG after annealing at $1273 \mathrm{~K}$ at the site of ${ }^{111} \mathrm{In}\left({ }^{111} \mathrm{Cd}\right)$ in $\mathrm{GaN}$ at different sample temperatures yielded a surprising result (Fig. 6) [29]. In order to describe the data, it is necessary to assume two fractions of probe nuclei situated in different environments. At $293 \mathrm{~K}$ (Fig. $6 \mathrm{~b}$ ), the larger fraction $\left(\mathrm{f}_{\mathrm{S}}\right.$ ) has a value of $65(5) \%$ and is characterized by $v_{Q}^{S}=6.3(1) \mathrm{MHz}$ with $\eta=0$. Further, the observed pattern is consistent with an orientation of $V_{z z}$ parallel to the $<0001>$ axis of the crystal. The second fraction $\mathrm{f}_{\mathrm{D}}=35(5) \%$ cannot be described by a unique interaction but shows a broad Lorentzian distribution of quadrupole interaction frequencies centered around $v_{Q}^{D}=17(3) \mathrm{MHz}(\eta=0)$. 

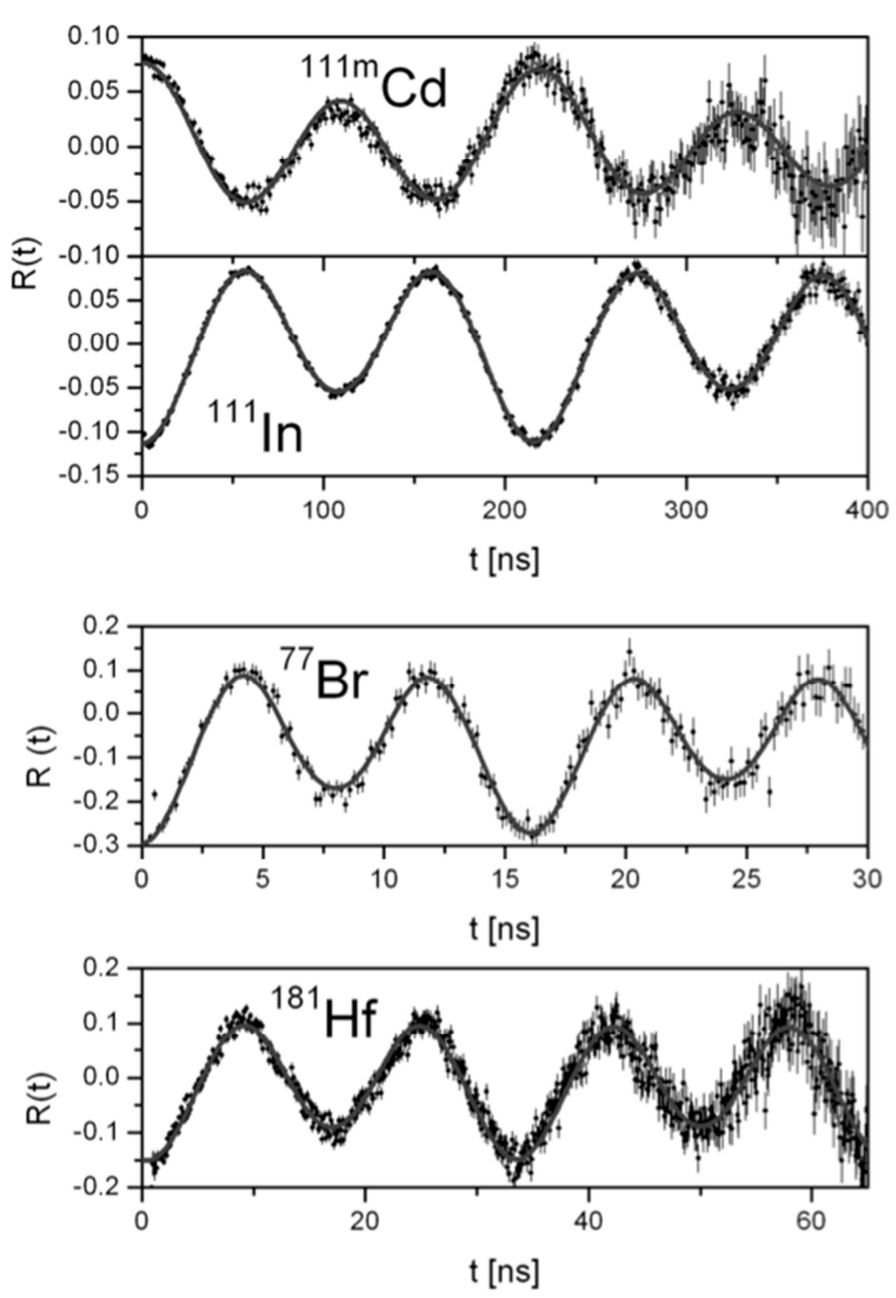

Fig. 5: PAC spectra for different probe atoms in $\mathrm{ZnO}$. All samples are annealed at $1300 \mathrm{~K}$ in nitrogen.
If the sample is cooled below room temperature, the fractions $f_{S}$ and $f_{D}$ remain constant. $v_{Q}^{S}$ decreases slowly (Fig. 6 a) and reaches a minimum value of $6.0(1) \mathrm{MHz}$ at $16.5 \mathrm{~K}$. At the same time, the mean value $v_{Q}^{D}$ of the second fraction increases steeply and reaches a value of $v_{Q}^{D}=140(11) \mathrm{MHz}$ at $16.5 \mathrm{~K}$. This leads to the sharp drop at the beginning of the observed spectra. For temperatures above room temperature, slightly higher values for $v_{Q}^{S}$ are obtained from the fit. $v_{Q}^{D}$ and especially the width of this frequency distribution decreases with temperature. Above $550 \mathrm{~K}$, the two fractions become indistinguishable and the whole spectrum can be described by only one fraction $\mathrm{f}_{\mathrm{S}}=100 \%$ showing only $v_{Q}^{S}$ (Fig. 6 c), which subsequently rises to a value of $9.2(1) \mathrm{MHz}$ at $1023 \mathrm{~K}$. These results are well reproducible for different samples and the temperaturedependent variation of $f_{S}$ (Fig. 7) and $f_{D}$ as well as $v_{Q}^{S}$ and $v_{Q}^{D}$ are fully reversible as long as the sample is not damaged by nitrogen evaporation from the surface. Such irreversible damage could only be detected in samples held for more than $8 \mathrm{~h}$ at $1273 \mathrm{~K}$.

A similar behavior is found for In in AlN [20]. After annealing, at $293 \mathrm{~K}$ two structures are clearly distinguishable in the PAC spectrum (Fig. 8a). One modulation is extending over the whole time range and can be described by a fraction $\mathrm{f}_{\mathrm{S}}=26.6(8) \%$ of ${ }^{111}$ In probes with a quadrupole interaction frequency $v_{Q}^{S}=27.0(3) \mathrm{MHz}(\eta=0, \delta=3.0(3) \%)$. A second interaction frequency, visible only in the beginning of the spectrum ( $\leq 50 \mathrm{~ns})$ is much larger, $v_{Q}^{X}=308(3) \mathrm{MHz}$, again axially symmetric $(\eta=0, \delta=10.0(5) \%)$, and reaches a fraction $f_{X}=42.4(9) \%$. Further, it is necessary to assume a third fraction $\mathrm{f}_{\mathrm{D}}$ of probe nuclei situated in an environment similar to the first but producing an axially asymmetric EFG. It is characterized by $v_{Q}^{D}=31.7(3) \mathrm{MHz}(\eta=0.5(1), \delta=$ $7.6(8) \%)$ and $\mathrm{f}_{\mathrm{D}}$ reaches a value of $31(1) \%$ at $293 \mathrm{~K}$.

In order to derive the relation of $V_{z z}$, the principal axes of the EFGs, to the crystal lattice, the $<0001>$ axis of the sample was oriented in three different directions relative to the detectors. The changes in the spectra observed for the different orientations provide a textbook example of an EFG orientation measurement (Fig. 8a-c) [1]. Alignment of the <0001> axis with the angel bisector of two adjacent detectors yields the double base frequency, perpendicular to the detector plane only the base frequency is observed and if the <0001> axis is pointed towards one detector the 
interaction frequency vanishes completely. The fits perfectly agree with this assumption that both interactions, $v_{Q}^{S}$ and $v_{Q}^{X}$, are due to EFGs with their principal component $V_{z z}$ aligned with the <0001>

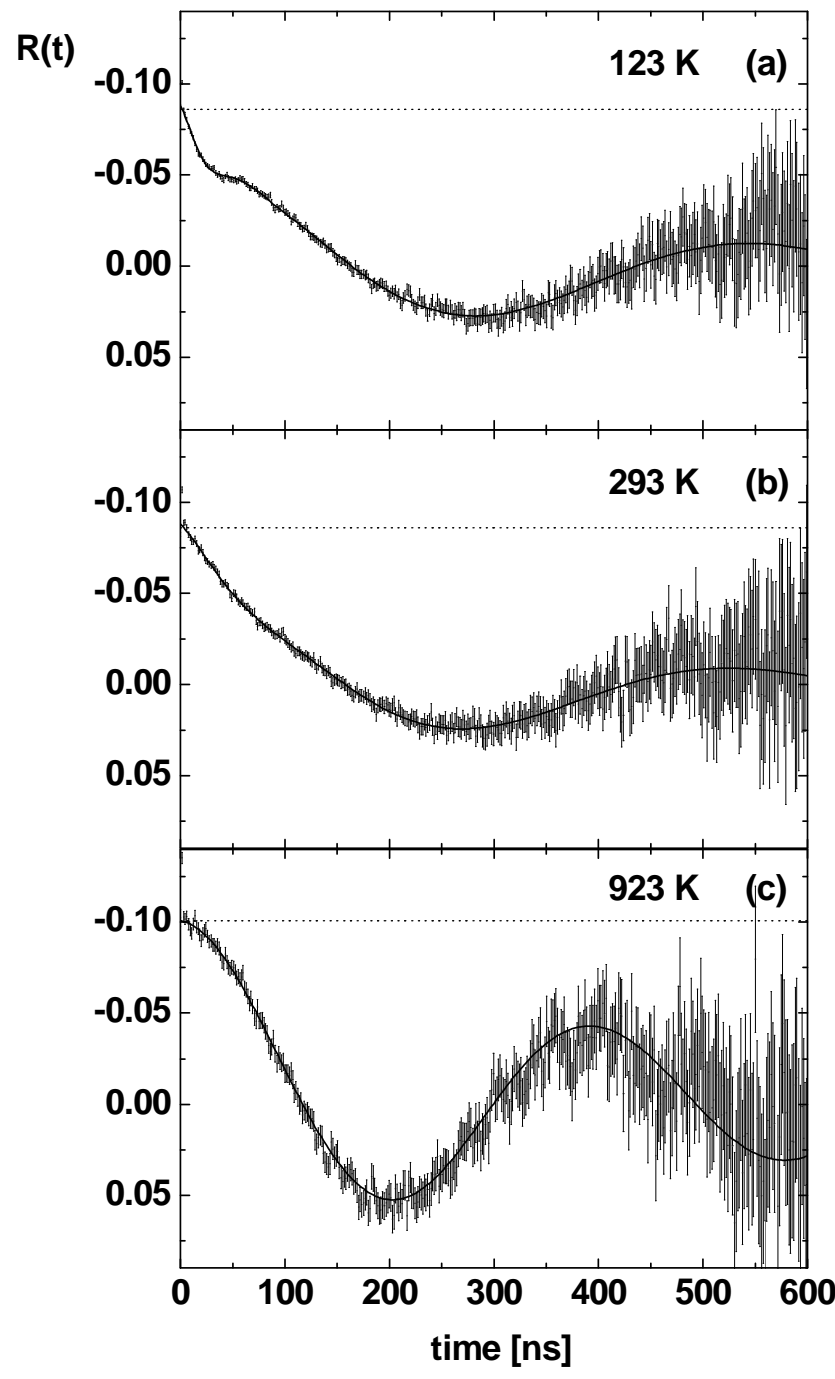

Fig. 6: PAC spectra obtained with ${ }^{111}$ In in $\mathrm{GaN}$ after implantation and annealing at different sample temperatures as indicated in the frames. The <0001> axis of the crystal was aligned with the angle bisector of two adjacent detectors.

crystal axis. The slight reduction of the anisotropy with time, most clearly visible when the <0001> axis is oriented towards one detector (Fig. $8 \mathrm{c})$, is due to the small distributions present in all frequencies and the influence of probes $\left(f_{D}\right)$ subjected to the axially asymmetric EFG.

As described above it is known that In implanted into $\mathrm{AlN}$ is incorporated to more than $\sim 90 \%$ on substitutional Al-sites in agreement with electron emission channeling (EC) measurements [44]. Therefore, it will be assumed that practically all PAC probes $\left(f_{S}+f_{D}+f_{X}\right)$ are situated on substitutional $\mathrm{Al}$ sites.

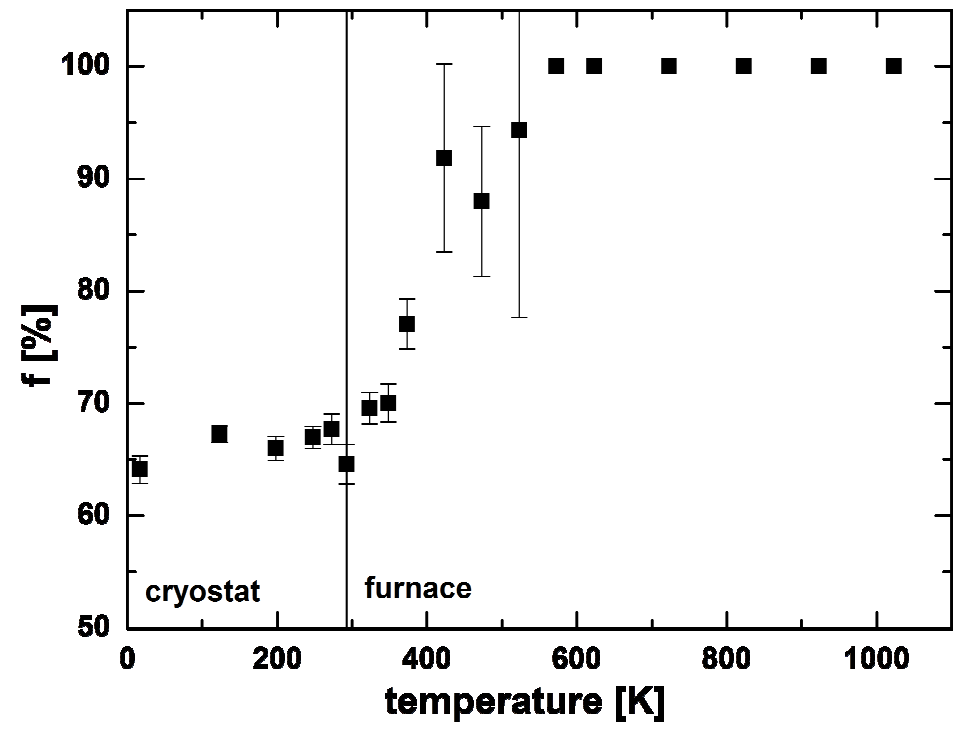

Fig. 7: Fraction of ${ }^{111} \mathrm{In}$ probes experiencing the $\mathrm{GaN}$ lattice EFG at different temperatures in a fully annealed sample. 
The orientation, symmetry and magnitude of the EFG experienced

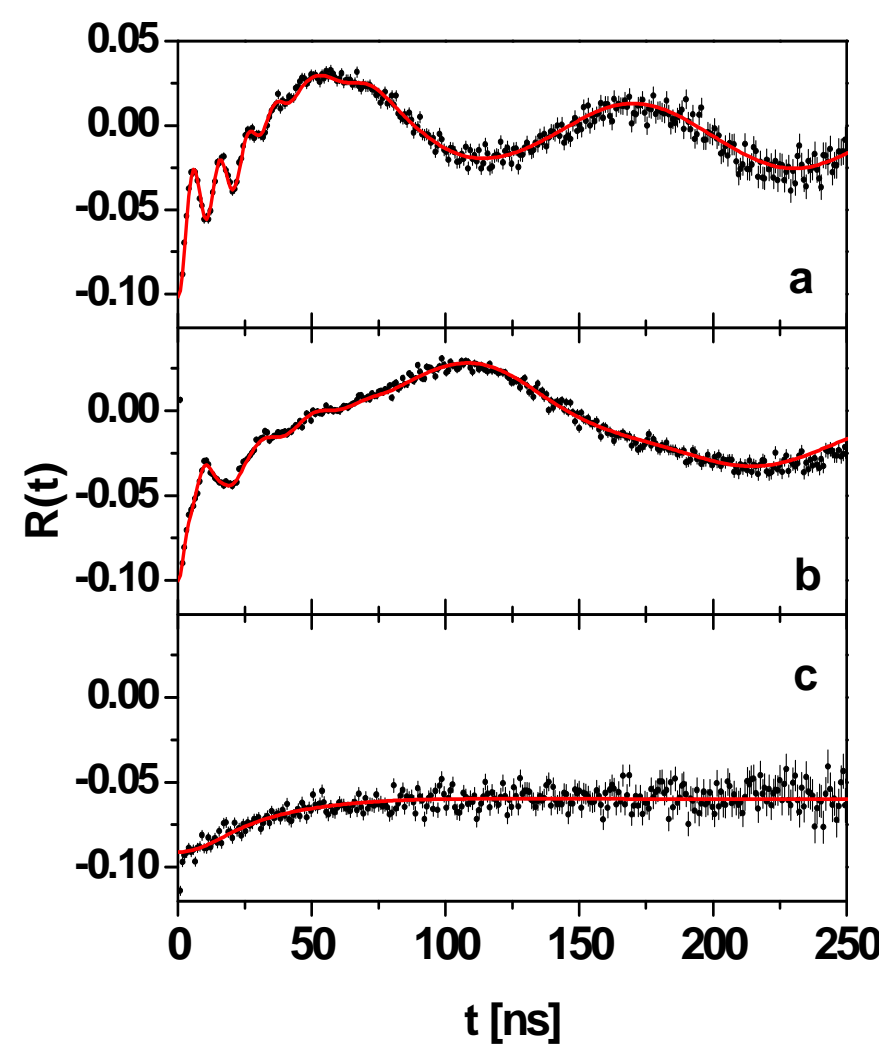

Fig. 8: PAC spectra obtained with ${ }^{111}$ In in AlN at $293 \mathrm{~K}$ for different orientations of the <0001> axis relative to the detectors. (a) aligned with the angle bisector between two detectors at $90^{\circ}$, (b) perpendicular to the detector plane, (c) towards one detector. The solid lines represent fits to the experimental data.

by fraction $\mathrm{f}_{\mathrm{S}}$ agree well with the symmetry properties of an $\mathrm{Al}$ site with an undisturbed surrounding in the wurtzite lattice structure. Therefore, we assign this fraction to In probes on undisturbed Al lattice sites (Pos. 1 in Fig. 2)

Of the two remaining fractions, $f_{X}$ is subjected to an axially symmetric EFG, of much larger magnitude $\left(v_{Q}^{X}=308(3) \mathrm{MHz}\right)$. Such values are typically observed in metals and semiconductors when Indium probes are associated with a nearest neighbor defect [45]. This fast frequency is present in all measurements after annealing above $700 \mathrm{~K}$, presumably the temperature at which a point defect becomes mobile and gets trapped at the probe. Substitutional In, being much larger than $\mathrm{Al}\left(0.53 \AA\right.$ for $\mathrm{Al}^{3+}$ versus $0.76 \AA$ for $\mathrm{In}^{3+}$ in a 4-fold coordinated environment [46]), creates a local strain field, which represents an attractive potential for diffusing defects, especially the nitrogen vacancy. Since each In probe is surrounded by four nitrogen atoms one would normally expect four trapping sites which cause EFGs with different orientations of their principal component. However, in the AlN Wurtzite lattice the vacancy situated along the $\langle 0001\rangle$ axis (Pos. 3 in Fig. 2) is slightly closer to the In probe so that trapping a $V_{N}$ in this position is energetically more favorable explaining why only one EFG with its principal component $V_{z z}$ oriented along the $<0001>$ axis is observed in the experiments. This picture also agrees well with the RBS/C observations described above showing that the displacement of In from the perfect substitutional Al-site is higher for the tilted axis than the c-axis. A probable displacement of In along the <0001> axis towards the nitrogen vacancy is only visible in channeling scans across tilted axes.

Assuming an abundance of $V_{N}$ in the sample, also the third fraction $f_{D}$ can be readily explained in this picture as probes which trap $\mathrm{V}_{\mathrm{N}}$ in a second or third nearest neighbor shell. Since the distance from the probe is much larger than for fraction $f_{X}$ the resultant EFG is only slightly larger than for an undisturbed probe but it will cause an asymmetry when $V_{N}$ is not trapped along the direction of the <0001> axis. Indeed, simple calculations of the lattice field gradient $V_{z z}^{\text {latt }}$ using the point charge model [47] reproduce reasonably well the magnitude and asymmetry parameter for all 
three fractions. For $\operatorname{In}(\mathrm{Cd})$ on an undisturbed $\mathrm{Al}$ site a value of $V^{P C}=1.8 \times 10^{15} \mathrm{~V} / \mathrm{cm}^{2}(\eta=0)$ is obtained and for $\operatorname{In}(\mathrm{Cd})$ with a nitrogen vacancy in the nearest neighbor position along the <0001>

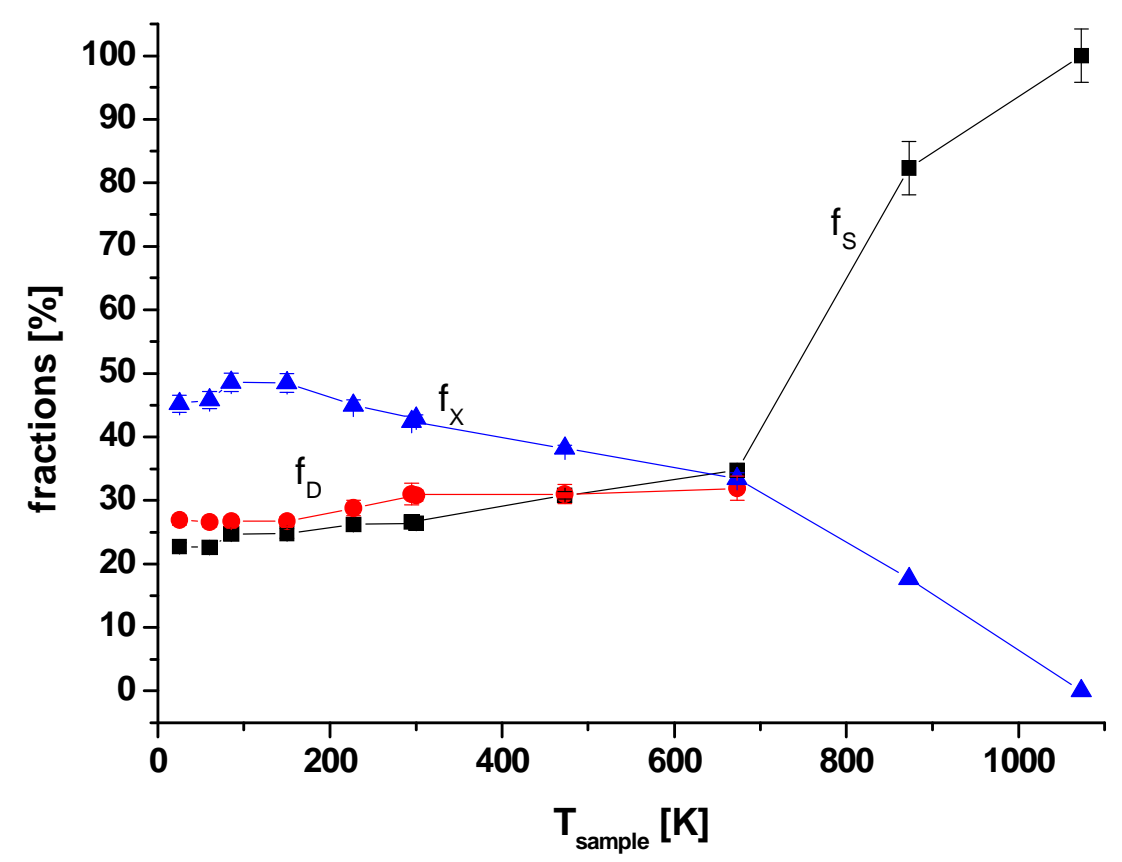

Fig. 9: Fractions of ${ }^{111}$ In probes in an AlN lattice experiencing different EFGs at different temperatures in a fully annealed sample.

axis the model yields $V_{z z}^{P C}=44 \times 10^{15} \mathrm{~V} / \mathrm{cm}^{2}(\eta=0)$. The values derived from the experiment are $V_{z z}^{\text {exp }}=4.8(1) \times 10^{15} \mathrm{~V} / \mathrm{cm}^{2}(\eta=0)$ (see Tab. 2$)$ and $V_{z z}^{\text {exp }}=54(1) \times 10^{15} \mathrm{~V} / \mathrm{cm}^{2}(\eta=0)$, respectively.

Measurements at elevated temperatures (Fig. 9) result in slightly higher values for $v_{Q}^{S}$, which reaches $34.4(3) \mathrm{MHz}$ at $1073 \mathrm{~K}$. In contrast to that, $v_{Q}^{X}$ drops very rapidly and assumes the same value as $v_{Q}^{S}$ at $1073 \mathrm{~K} . v_{Q}^{D}$ rises slowly like $v_{Q}^{S}$, its asymmetry parameter drops, and above $700 \mathrm{~K}$ it becomes indistinguishable from $v_{Q}^{S}$. Thus, at the highest temperature the whole spectrum can be described by only one fraction with $v_{Q}^{S}=34.4(3) \mathrm{MHz}$. Most surprisingly as in GaN this variation is completely reversible. After keeping the sample for several hours at $1073 \mathrm{~K}$ a subsequent measurement at $293 \mathrm{~K}$ could be described with exactly the same parameters as before.

The variation of the interaction frequencies as well as their fractions with sample temperature is surprising. When measured with the transition metal ${ }^{181} \mathrm{Hf}_{\mathrm{Al}}$ in $\mathrm{AlN}$ as a probe [48] the EFG for undisturbed substitutional probes only changes by approximately $3 \%$ in the temperature range whereas we observe a $23 \%$ increase of the EFG measured with ${ }^{111} \mathrm{In}$. The EFG was found to decrease when measured with the ${ }^{181} \mathrm{Hf}$ probe proving that thermal lattice expansion cannot be the sole reason for the change of the EFG with temperature. It is known that the lattice parameters of nitrides can change by incorporation of dopants or impurities and considerable hydrostatic strain can be introduced [49]. However, the relative change of the lattice parameters with temperature should be comparable for Hf and In impurities. Therefore, the large increase between the value of $v_{Q}^{S}$ at 293 and at $1073 \mathrm{~K}$ hints towards a special behavior of In in AlN and similarly for GaN [33]. 
The variation of $v_{Q}^{X}$ and $f_{X}$ with temperature is even more astonishing. The quick drop of $v_{Q}^{X}$ from

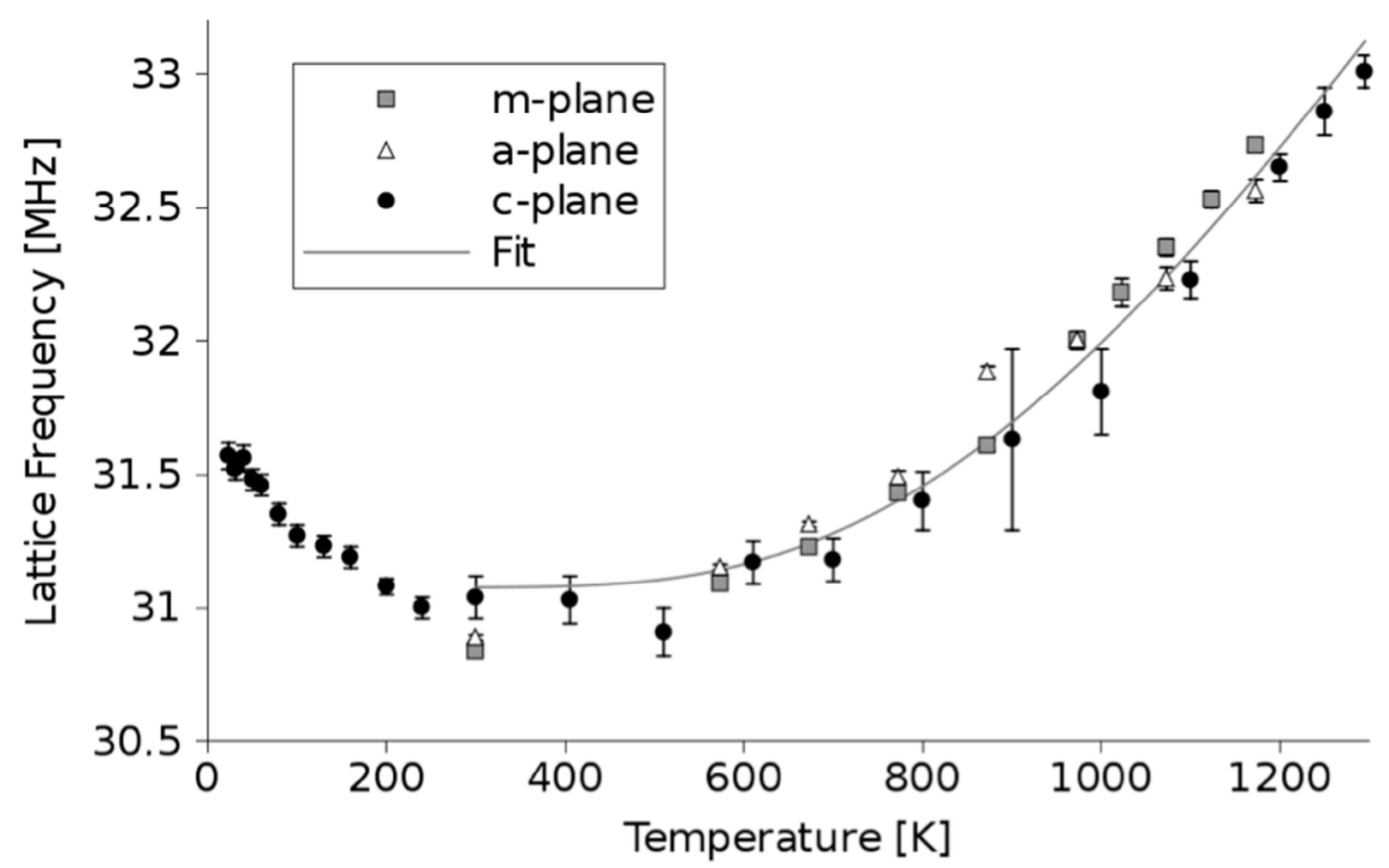

Fig. 10: Temperature dependence of the lattice-EFG in $\mathrm{ZnO}$. The temperatures above $300 \mathrm{~K}$ can be described by a model suggested by Wolf et. al. [37] (Solid line). Three differently oriented crystal surfaces have been implanted: a-plane, m-plane and c-plane, but no effect due to different damage susceptibilty could be observed. c-plane data are taken from ref. [23].

$308 \mathrm{MHz}$ at $293 \mathrm{~K}$ to $34.4 \mathrm{MHz}$ at high temperatures, where it becomes undistinguishable from $v_{Q}^{S}$, indicates a basic change in the vicinity of the probe. This can either be due to a shift in the position of the probe relative to the ideal $\mathrm{Al}$ lattice site and/or the disappearance of a nearest neighbor (NN) defect. At present we favor a model featuring both elements. At room temperature a nitrogen vacancy is trapped in the $<0001>$ nearest neighbor position next to the In probe, which is also displaced slightly towards the vacancy. With increasing temperature the mean position of the probe approaches the ideal Al lattice position causing a rapid drop of $v_{Q}^{X}$. Above $700 \mathrm{~K}$ only the EFG due to the undisturbed wurtzite lattice is observed, i.e. the probe - defect complex broke up. The fact that the complex is again observed at room temperature can be understood by looking at the details of the PAC measurement. Prior to the start of the $\gamma-\gamma$ cascade ${ }^{111}$ In decays to an excited state of ${ }^{111} \mathrm{Cd}$ with a half-life of $\mathrm{t}_{1 / 2}=0.12 \mathrm{~ns}$. It seems that above $700 \mathrm{~K}$ the $\mathrm{Cd}-\mathrm{V}_{\mathrm{N}}$ complex is no longer stable and the $\mathrm{NN}$ vacancy disappears within this time interval. However, all $\mathrm{In}-\mathrm{V}_{\mathrm{N}}$ complexes where In does not decay during the measurement remain stable and thus the $v_{Q}^{X}$ can again be observed with the same amplitude $f_{X}$ as before.

A similar process can explain the results found in GaN although the QIF induced by the defect is not as unique as in AlN.

The temperature variation of the EFG at the site of $\mathrm{In}$ in $\mathrm{ZnO}$ is considerably weaker than in the previously discussed nitrides (Fig. 10). In contrast to the previous systems here no change in the 
site fraction is observed. At low temperatures, the QIF decreases from $31.6(4) \mathrm{MHz}$ at $10 \mathrm{~K}$ to 31.0 (4) $\mathrm{MHz}$ at room temperature and increases to $33.8(4) \mathrm{MHz}$ at $1420 \mathrm{~K}$ [37]. Like in the nitrides, the temperature dependence cannot be described by the change of the lattice constants or a negative $\mathrm{T}^{3 / 2}$ dependence, which was found for metals [50]. Wolf et al. [37] suggested that the temperature dependence can be explained by an increasing electron density caused by the appearance of donors related to interstitial $\mathrm{Zn}$ with an activation enthalpy of $0.4 \mathrm{eV}$. However, recent theoretical calculations by Erhart et al. [51] cast serious doubts onto this model, since for $\mathrm{Zn}_{\mathrm{i}}$ a formation enthalpy of $2.7 \mathrm{eV}$ is obtained.

\section{Conclusions}

The recovery of lattice damage due to the implantation of various impurities into group III nitride semiconductors and $\mathrm{ZnO}$ has been studied by PAC and RBS/Channelling. Except for In in $\mathrm{GaN}$ and $\mathrm{AlN}$, an almost complete annealing of the damage was found after annealing between 1000 and $1300 \mathrm{~K}$. Generally, $\mathrm{ZnO}$ was found to incorporate implanted impurities much more readily than the nitride semiconductors. A comparison of the magnitude of the electric field gradient determined after the highest annealing step to the values derived from NQR measurements for the pure systems $[52,53]$ (Table 2) indicates that in all cases the impurities are incorporated substitutionally onto the cation site. This conclusion is supported by lattice location measurements available in the literature and RBS/Channelling measurements carried out in the course of this study.

A very interesting behavior was found for $\mathrm{In}$ in GaN and AlN. The relatively small substitutional fraction measured after implantation of $\mathrm{In}$ in $\mathrm{GaN}$ and $\mathrm{AlN}$ grew to $100 \%$ at elevated sample temperatures and dropped back again to the lower value when the temperature was reduced to room temperature. The well-defined EFG associated with the non-substitutional fraction of In probes is explained by assuming that a stable complex between In and a nitrogen vacancy is formed. This complex might be the radiative trapping centre for excitons which could explain the large quantum efficiency of $\mathrm{Ga} /(\mathrm{Al}) \mathrm{InN}$ devices.

\section{Acknowledgment}

KL thanks FCT, Portugal for support (Ciência 2007, PTDC/FIS/65233/2006, and PTDC/CTM/100756/2008).

\section{References}

[1] M. Zacate and H. Jaeger: present volume

[2] Ioffe Institute, St. Petersburg, http://www.ioffe.rssi.ru/SVA/NSM/

[3] E.H. Kisi and M.M. Elcombe: Acta Cryst. C Vol. 45 (1989), p. 1867

[4] J.E. Jaffe and A.C. Hess: Phys. Rev. B Vol. 48 (1993), p. 7903

[5] L. Gerward and J.S. Olsen: J. Synchrotron Radiat. Vol. 2 (1995), p. 233

[6] D.R. Lide (Ed.): CRC Handbook of Chemistry and Physics, 73rd Edition (CRC Press, New York, 1992).

[7] S. Adachi, Properties of Group-IV, III-V and II-VI Semiconductors (John Wiley and Sons, Ltd, West Sussex, England, 2005).

[8] K. P. O'Donnell, R. W. Martin, and P. G. Middleton: Phys. Rev. Lett. Vol. 82 (1999), p. 237 
[9] T. Onuma, A. Chakraborty, B. A. Haskell, S. Keller, S. P. DenBaars, J. S. Speck, S. Nakamura, U. K. Mishra, T. Sota, and S. F. Chichibu: Appl. Phys. Lett. Vol. 86 (2005), p. 151918

[10] F. A. Ponce, S. Srinivasan, A. Bell, L. Geng, R. Liu, M. Stevens, J. Cai, H. Omiya, H. Marui, S. Tanaka: Phys. Status Solidi B Vol. 240 (2003), p. 273

[11] A. Hangleiter, F. Hitzel, C. Netzel, D. Fuhrmann, U. Rossow, G. Ade, and P. Hinze: Phys. Rev. Lett. Vol. 95 (2005), p. 127402.

[12] S. F. Chichibu, A. Uedono, T. Onuma, B. A. Haskell, A. Chakraborty, T. Koyama, P. T. Fini, S. Keller, S. P. DenBaars, J. S. Speck, U. K. Mishra, S. Nakamura, S. Yamaguchi, S.

Kamiyama, H. Amano, I. Akasaki, J. Han, and T. Sota: Nature Materials Vol. 5 (2006), p. 810

[13] V. Kachkanov, K. P. O’Donnell, S. Pereira, R. W. Martin: Philosophical Magazine Vol. 87 (2007), p. 1999

[14] C. Ronning, M. Dalmer, M. Deicher, M. Restle, M.D. Bremser, R.F. Davis, H. Hofsäss: Mat. Res. Soc. Symp. Proc. Vol. 468 (1997), p. 407

[15] A. Burchard, E.E. Haller, A. Stötzler, R. Weissenborn, M. Deicher, and the ISOLDE Collaboration: Physica B Vol. 273/274 (1999), p. 96

[16] K. Lorenz, F. Ruske, and R. Vianden: Phys. Stat. Sol. (b) Vol. 228 (2001), p. 331

[17] J. Bartels, K. Freitag, J.G. Marques, J.C. Soares and R. Vianden: Hyperfine Interact. Vol. 120/121 (1999), p. 397

[18] K. Lorenz, PhD thesis, University of Bonn (2002)

[19] K. Lorenz, R. Vianden: Phys. Stat. Sol. (c) Vol. 1 (2002), p. 413

[20] J. Schmitz, J. Niederhausen, J. Penner, K. Lorenz, E. Alves, R. Vianden: Physica B Vol. 404 (2009), p. 4866

[21] P. J. M. Smulders, D.O. Boerma: Nucl. Instrum. Methods Phys. Res. B Vol. 29 (1987), p. 471

[22] R. Dogra, S.K. Shrestha, A.P. Byrne, M.C. Ridgway, A.V.J. Edge, R. Vianden, J. Penner, and H. Timmers: J. Phys.: Condens. Matter Vol. 17 (2005), p. 6037

[23] D. Forkel, PhD thesis, University of Erlangen (1987)

[24] F.D. Feiock and W.R. Johnson: Phys. Rev. Vol. 187 (1969), p. 39

[25] R. M. Sternheimer: Bull. Amer. Phys. Soc. Vol. 12 (1967), p. 108

[26] H. Granzer, H. H. Bertschat, H. Haas, W.-D. Zeitz, J. Lohmüller, and G. Schatz: Phys. Rev. Lett Vol. 77 (1996), p. 4261

[27] Value for $\mathrm{Br}^{-}$taken from E.G. Wikner and T.P. Das: Phys. Rev. B Vol. 109 (1958), p. 360

[28] P. Herzog, K. Freitag, M. Reuschenbach, and H. Walitzki: Z. Phys. A Vol. 294 (1980), p. 13.

[29] K. Lorenz, F. Ruske, and R. Vianden: Appl. Phys. Lett. Vol. 80 (2002), p. 4531

[30] C.M. Lederer and V.A. Shirley (Ed.): Table of Isotopes 7th edition (J. Wiley, New York, 1980).

[31] M. Dietrich, M. Deicher, A. Stotzler, R. Weissenborn and the ISOLDE collaboration: Nucl. Phys. A Vol. 701 (2002), p. 240 
[32] T. Butz and A. Lerf: Phys. Lett. Vol. 97A (1987), p. 217

[33] K. Lorenz, T. Geruschke, E. Alves and R. Vianden: Hyperfine Interact., Vol. 177 (2007), p. 89

[34] R. Nédélec, R. Vianden and the ISOLDE Collaboration: Hyperfine Interact. Vol. 178 (2007), p. 19

[35] R.E.J. Sears: Phys. Rev. B Vol. 22 (1980), p. 1135

[36] Woo-Sik Jung, Oc Hee Han, Seen-Ae Chae: Materials Letters Vol. 61 (2007), p. 3413

[37] H. Wolf, S. Deubler, D. Forkel, H. Foettinger, M Iwatschenko-Borho, F. Meyer, M. Renn, W. Witthuhn, and R. Helbig: Materials Science Forum, Vol. 10-12 (1986), p. 863

[38] S. Deubler, J. Meier, R. Schütz, and W. Witthuhn : Nuc. Instr. Meth. B Vol. 63 (1992), p. 223

[39] R. Dogra, A.P. Byrne, M.C Ridgway: Optical Materials Vol. 31 (2009), p. 1443

[40] E. Rita, J. G. Correia, U. Wahl, E. Alves, A.M.L. Lopes, and J.C. Soares, and the ISOLDE collaboration: Hyperfine Interact. Vol. 158 (2004), p. 395

[41] This work

[42] Th. Geruschke, Bonn, private communication

[43] R. Nédélec, R. Vianden, and the ISOLDE Collaboration : Optical Materials Vol. 28 (2006), p. 723

[44] C. Ronning, M. Dalmer, M. Uhrmacher, M. Restle, U. Vetter, L. Ziegeler, H. Hofsäss, T. Gehrke, K. Järrendahl, R. F. Davis, and the ISOLDE Collaboration : J. Appl. Phys. Vol. 87 (2000), p. 2149

[45] R. Vianden in Nuclear Physics Applications in Materials Science edited by. E. Recknagel and J.C. Soares, NATO ASI series, series E Vol. 144 (1988), p. 239, and Th. Wichert, Semiconductors and Semimetals Vol. 51B (1999), p. 297

[46] R.D. Shannon and C.T. Prewitt: Acta Cryst. B Vol. 25 (1969), p. 925

[47] F. De Wette: Phys. Rev. Vol. 123 (1961), p. 103

[48] K. Lorenz and R. Vianden: Hyperfine Interact. Vol. 158 (2004), p. 273

[49] C. Kisielowski, J. Krüger, S. Ruvimov, T. Suski, J. W. Ager III, E. Jones, Z. Liliental-Weber, M. Rubin, E. R. Weber, M. D. Bremser, and R. F. Davis: Phys. Rev. B Vol. 54 (1996), p. 17745

[50] J. Christiansen, P. Heubes, R. Keitel, W. Klinger, W. Loeffler, W. Sandner, and W. Witthuhn: Z. Phys. B Vol. 24 (1976), p. 177

[51] P. Erhart N. Juslin, O. Goy, K. Nordlund, R. Müller, and K. Albe, J. Phys.: Condens. Matter Vol. 18 (2006), p. 6585

[52] G. Denninger and D. Reiser: Phys. Rev B Vol. 55 (1997), p. 5073

[53] M. Corti, A. Gabetta, M. Fanciulli, A. Svane, and N. E. Christensen: Phys. Rev B Vol. 67 (2003), p. 64416 